\title{
Hadron spectra from overlap fermions on HISQ gauge configurations.
}

\section{S. Basak ${ }^{a}$, S. Datta ${ }^{b}$, A. T. Lytle ${ }^{b}$, Padmanath M. ${ }^{b}$, P. Majumdar ${ }^{c}$, and N. Mathur ${ }^{* b}$ (Indian Lattice Gauge Theory Initiative)}

${ }^{a}$ School of Physical Sciences, National Institute of Science Education and Research, Bhubaneswar 751 005, India

${ }^{b}$ Department of Theoretical Physics, Tata Institute of Fundamental Research, Homi Bhabha Road, Mumbai 400005, India

${ }^{c}$ Department of Theoretical Physics, Indian Association for the Cultivation of Science, Kolkata 700032, India.

E-mail: nilmani@theory.tifr.res.in

Adopting a mixed action approach, we report here results on hadron spectra containing one or more charm quarks. We use overlap valence quarks on a background of $2+1+1$ flavor HISQ gauge configurations generated by the MILC collaboration. We also study the ratio of leptonic decay constants, $f_{D_{s}^{*}} / f_{D_{s}}$. Results are obtained at two lattice spacings.

31st International Symposium on Lattice Field Theory LATTICE 2013

July 29-August 3, 2013

Mainz, Germany

* Speaker. 


\section{Introduction}

Recently there has been a resurgence of interest in heavy hadron spectroscopy with the discovery of numerous hadrons with one or more heavy quarks. Results from LHC and future charmbottom factories are expected to add to the excitement in this field in the near future. However, the study of heavy hadrons using lattice QCD has an inherent problem since at these masses, with currently available lattices, the condition am $<<1$ in general is not satisfied which leads to larger systematic errors. Though NRQCD is successful in studying bottom quark it is not so clear whether one can use that to study hadrons with one or more charm quarks. Relativistic heavy quark actions, where all $O\left((\mathrm{am})^{n}\right)$ corrections are systematically removed, are becoming increasingly popular $[1,2]$.

In this work we have adopted a mixed action approach by the using overlap action [3] for valence quarks on a background of $2+1+1$ flavours HISQ gauge configurations [4]. The overlap action is automatically $\mathscr{O}(m a)$ improved; it also offers various simplifications in studies of decay constants. An aim of this study is to investigate the behavior of the overlap action in the regime $m a \lesssim 1$. The overlap action also has some desirable features computationally, such as the adaptation of multi mass algorithms [5]. However, using overlap action for the dynamical quarks is still prohibitively costly, except with fixed topology [6]. Therefore, for the gauge configurations we have used the large set of $2+1+1$ flavours configurations generated by the MILC lattice collaboration [4] with the one-loop, tadpole improved Symanzik gauge action and the highly improved staggered quark (HISQ) fermion action [7]. Taste violations in the HISQ action were found to be small [7]. A similar mixed action approach has been taken by the $\chi Q C D$ collaboration using overlap valence quarks on $2+1$ flavours dynamical domain wall gauge configurations [8].

In this report, we present our preliminary results on charm and strange hadron spectra as well as leptonic decay constants for $D_{s}$ and $D_{s}^{*}$ mesons, using the above-mentioned mixed action approach. This is an update of our ongoing study; earlier results were reported in Ref. [9].

\section{Numerical details}

We used two sets of dynamical $2+1+1$ flavours HISQ lattice ensembles, generated by the MILC collaboration : a set of $32^{3} \times 96$ lattices at gauge coupling $10 / \mathrm{g}^{2}=6.30$ and another set of $48^{3} \times 144$ lattices at $10 / g^{2}=6.72$. The strange and charm masses are set at their physical values while $m_{l} / m_{s}=1 / 5$ for both lattices. The details of these configurations are summarized in Ref. [4]. We determined the lattice spacing by equating the $\Omega$ (sss) baryon mass measured on these ensembles with its physical value. The strange mass was tuned by setting the $\bar{s} s$ pseudoscalar mass to $685 \mathrm{MeV}$ [10]. The measured lattice spacings are $0.0877(10)$ and $0.0582(5) \mathrm{fm}$ for $32^{3} \times 96$ and $48^{3} \times 144$ lattices respectively which are consistent with $0.0888(8)$ and $0.0582(4) \mathrm{fm}$ as measured by MILC collaboration by using $r_{1}$ parameter [4]. The results reported here were obtained from 110 configurations on the coarser lattice, and 65 configurations on the finer lattice.

For valence quarks we used overlap action [3]. For the numerical implementation of massive overlap fermions we followed the methods used by the $\chi Q C D$ collaboration [11]. The low Wilson eigenmodes are projected out by the Arnoldi method and the Zolotarev approximation is used to evaluate the sign function. We used the usual periodic boundary condition in the spatial and antiperiodic in the temporal directions. Gauge configurations were first fixed to Coulomb gauge and 
then smeared with single level of HYP blocking. Using both point and wall sources we calculated various point-point, wall-point as well as wall-wall correlators.

Since for the charm quark $m a$ is not very small, we need to be careful about discretization errors. The overlap action does not have $O(\mathrm{ma})$ errors. In order to estimate the size of discretization errors coming from higher orders of $m a$, we look at the energy-momentum dispersion relation of the $1 \mathrm{~S}$ charmonia. Expanding the energy momentum relation in powers of $\mathbf{p} a$, one can write, for $|\mathbf{p}|<<m_{0}, 1 / a$

$$
E(p)^{2}=M_{1}^{2}+\frac{M_{1}}{M_{2}} \mathbf{p}^{2}+O\left(\mathbf{p}^{4}\right)=M_{1}^{2}+\mathbf{p}^{2} c^{2} .
$$

Here $M_{1}$ is the pole or rest mass $E(0)$, and $M_{2}$ is called the kinetic mass $\left(M_{1} / c^{2}\right)$. The difference between $M_{1}$ and $M_{2}$ is one measure of $\mathscr{O}(m a)$ cutoff effects. As highlighted in Ref. [1] (in the socalled Fermilab interpretation), since $M_{2}$ controls the non-trivial physics of a heavy hadron system, in using a relativistic action for heavy quarks, one should use $M_{2}$ to measure the masses.

The charm mass is tuned by setting the spin-averaged $1 \mathrm{~S}$ state mass, $\left(m_{\eta_{c}}+3 m_{J / \psi}\right) / 4$, to its physical value, where we take into account the kinetic mass, as defined above, in the definition of mass. Previously [9] we tuned our charm mass with pole mass of mesons and showed that velocity of light $(c)$ is not closer to 1 which leads to $O(m a)^{2}$ errors. We calculated pseudoscalar meson mass at various external momenta $p^{2}=(2 \pi / L)^{2} n^{2}$, with $n \leq 2$. We use wall source at finite momenta by putting a phase factor in the wall so as to project to a particular momentum. This method is more suitable for improving signal in correlators with finite momenta [9]. In Fig. 1(a) we show $E(p)^{2}$ for various momenta for the pseudoscalar meson on our finer lattices. The green line is for the continuum dispersion relation, $E^{2}=m^{2}+p^{2}$, while the blue line is the fitted dispersion relation with $c=0.96(2)$. For coarser lattices we obtain $c=0.92(3)$.

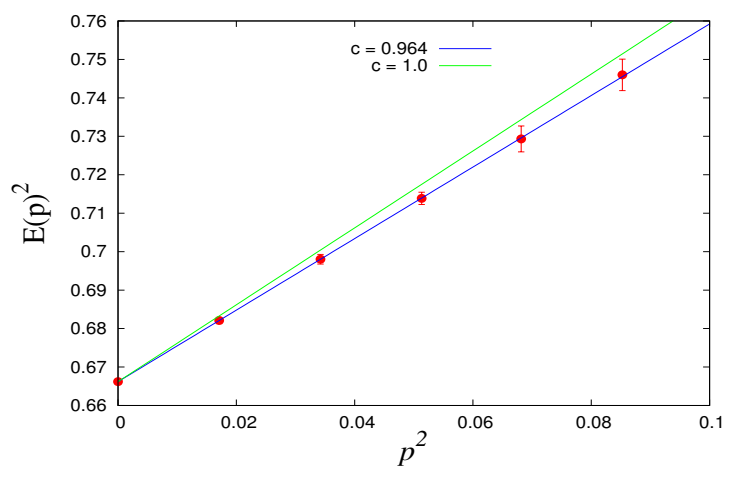

(a)

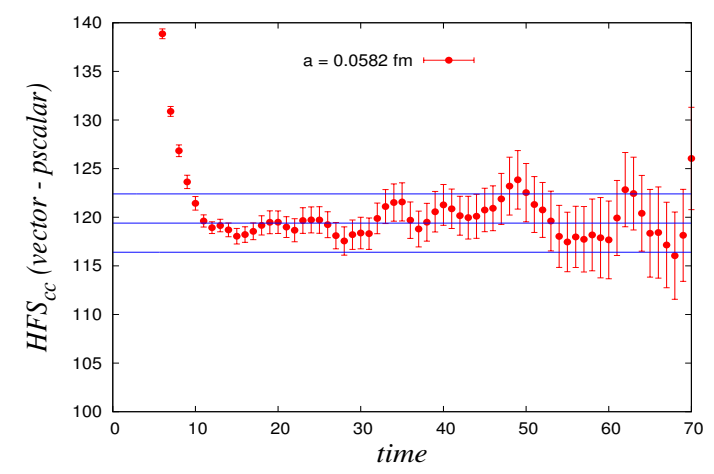

(b)

Figure 1: (a) Energy-momentum dispersion relation for the pseudoscalar meson at charm mass on the finer lattices. Blue line is with $c=0.96(2)$ obtained by fitting our data while green line is with $c=1$. (b) Effective hyperfine splitting in $1 \mathrm{~S}$ charmonia for wall-point correlators for lattices with spacing $0.0582 \mathrm{fm}$. Horizontal lines show the fit results with one sigma error.

\section{Results}

Multimass method help us to calculate the quark propagators over a wide range of quark masses with $10-12 \%$ overhead. Our extracted pseudoscalar meson masses are within the range $400-5130 \mathrm{MeV}$ and $230-4000 \mathrm{MeV}$ for the finer and coarser lattices respectively. In the following subsections we will discuss our results for mesons and baryons mainly in terms of energy splittings, as these have less systematic uncertainty as compared to extracted energies. 


\subsection{Hyperfine splitting in $1 S$ charmonia}

The hyperfine splitting in 1S charmonia is one of the most well studied physical quantities in lattice charmonium calculations over the years, and until very recently [12] lattice results were found to be smaller than the experimental value $(\sim 116 \mathrm{MeV})$. This underestimation is now understood to be mainly due to the discretization error associated with the charm quark action and the quenched approximation. In our study we calculated this splitting. In Fig. 1(b), we showed the effective splittings between vector and pseudoscalar correlators (jackknifed) at the tuned charm mass for wall-point correlators on finer lattices. Horizontal lines shown are the fit results, with one sigma errorbar. Our final estimated results, for this hyperfine splitting are 125(6) MeV and 119(3) $\mathrm{MeV}$ corresponding to coarser and finer lattices respectively.

\subsection{Energy splittings in charmonia and charmed-strange mesons}

Beside $1 \mathrm{~S}$ hyperfine splittings it is also important to consider energy splittings between various other charmonia. In Fig. 2(a) we plot energy splittings between axial, scalar and tensor charmonia from pseudoscalar charmonium. In addition to this, we also calculated charmed-strange mesons with various quantum numbers, and energy splittings between these mesons are also plotted in Fig. 2(a). It is to be noted that tuning the charm mass by using kinetic mass has brought these splittings closer to experimental values than those previously obtained in Ref. [9].

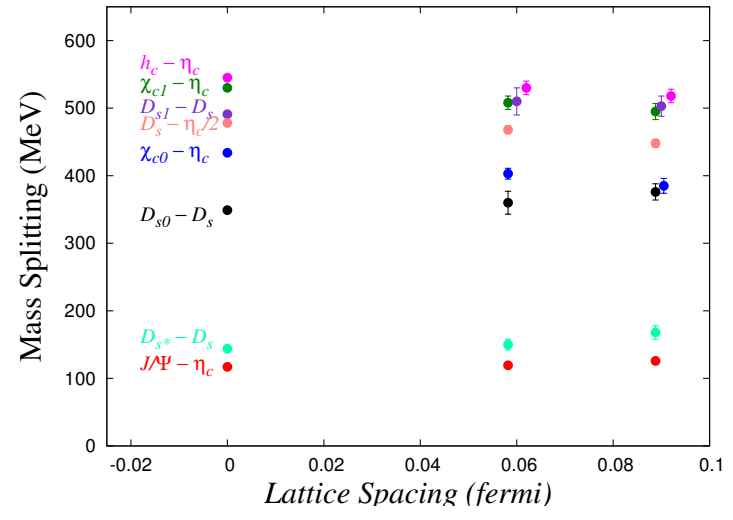

(a)

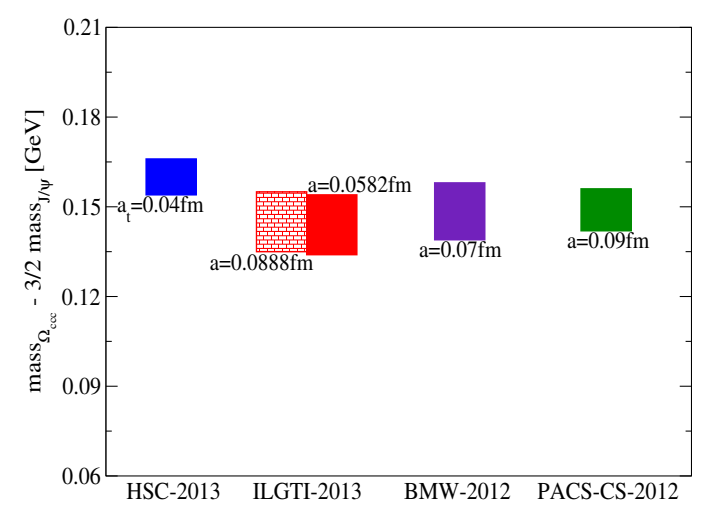

(b)

Figure 2: (a) Meson mass splitting for charmonia and charmed-strange mesons at two lattice spacings. Experimental values are shown in the left side. (b) The mass splitting of $\Omega_{c c c}-\frac{3}{2} J / \Psi$ along with other lattice results. Result from this work is shown in red colour.

\subsection{Charmed baryons}

Over the years the charmed mesons have been studied comprehensively and that provided us with invaluable understanding of the theory of strong interaction. However, the study of charmed baryons is still not comprehensive enough though that can also provide similar input. It is thus crucial to study these baryons by using a first principle method of lattice QCD. On our lattices we extracted ground state spectra of charmed baryons with one or more charm quark content, for example, baryons with quark content $c s u, c u u, c s s, c c s$, and $c c c$. The study of a particular baryon which draws immediate attention is the triply-charmed $\Omega(c c c)$, a baryon analogues of charmonia, which according to Bjorken [13], may provide a new window for understanding the structure of baryons. Though the theory of strong interaction unambiguously predict such a state, similar to 

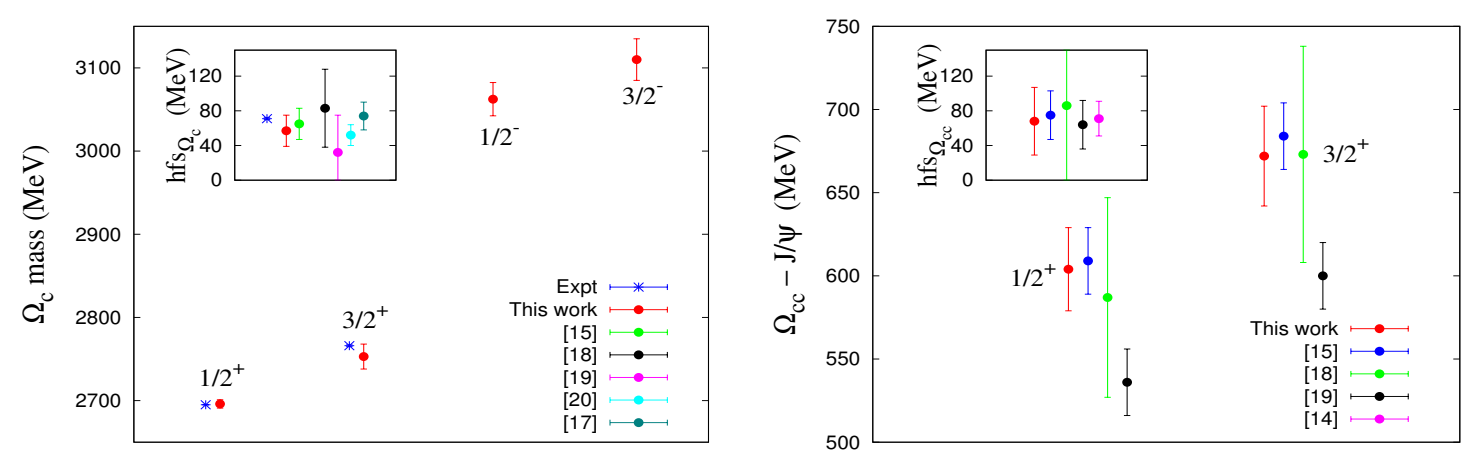

Figure 3: (a) $\Omega_{c}$ and (b) the mass splitting of $\Omega_{c c}-J / \Psi$. Inset figures are for hyperfine splittings between positive parity spin-3/2 and spin- $1 / 2$ states. Also shown are other lattice determinations, and the experimental values, where available.

its light quark counterpart $\Delta(и и u)$ and $\Omega(s s s)$, it has not been observed yet. In Fig. 2(b) we plot the mass splittings of $\Omega_{c c c}-\frac{3}{2} J / \Psi$. A factor $3 / 2$ is included to account for the difference in the charm quark content in $\Omega_{c c c}$ and $J / \Psi$, and thus this splitting mimics the binding energy for such a state. We also plotted other lattice determinations $[14,15,16]$ for this quantity and our result is consistent with those. In Fig. 3 we showed results for $\Omega_{c}(c s s)$ and $\Omega_{c c}(c c s)$ baryons, and for the later case energy splittings of $\Omega_{c c}-J / \Psi$. It is to be noted that for these baryons we extracted masses for both spin $1 / 2$ and spin $3 / 2$ with both parities, some of which are yet to be measured experimentally. In the inset we also plot the hyperfine splittings between positive parity spin-3/2 and spin-1/2 states. Our results are consistent with other lattice results $[15,17,18,19,20]$, and the experimental values, where available. We are in the process of adding another lattice spacing and after that we will we will carry out both continuum as well as chiral extrapolations by using mixed action partially quenched chiral perturbation theory [21]. One also needs to evaluate $\Delta_{\text {mix }}$ [22], the low energy constant representing $\mathscr{O}\left(a^{2}\right)$ discretization dependence.

\subsection{Decay constants}

The decay constants of heavy-light mesons are experimentally very important: they are essential ingredients in extracting CKM matrix elements from decays of heavy-light mesons. Here we present preliminary results for the pseudoscalar decay constant $f_{D_{s}}$, as well as the ratio of vector to pseudoscalar decay constants $f_{D_{s}^{*}} / f_{D_{s}}$. The decay constants $f_{D_{s}}$ and $f_{D_{s}^{*}}$ are defined by

$$
\left\langle 0\left|A_{\mu}\right| D_{s}(p)\right\rangle=f_{D_{s}} p^{\mu}, \quad\left\langle 0\left|V_{\mu}\right| D_{s}^{*}(p, \lambda)\right\rangle=f_{D_{s}^{*}} M_{D_{s}^{*}} \varepsilon_{\mu}^{\lambda}
$$

where $\varepsilon_{\mu}^{\lambda}$ is a polarization vector, and $A_{\mu}$ and $V_{\mu}$ are the continuum currents related to lattice operators by $\left\{A_{\mu}, V_{\mu}\right\}=\left\{Z_{A} \bar{s} \gamma_{\mu} \gamma_{5} c, Z_{V} \bar{s} \gamma_{\mu} c\right\}$. The $D_{s}$ decay constant is determined from the relation

$$
M_{D_{s}}^{2} f_{D_{s}}=\left(m_{c}+m_{s}\right)\left\langle 0|P| D_{s}\right\rangle,
$$

where $P=\bar{s} \gamma_{5} c$. Note that the quantities on the right are bare quantities.

We are currently in the process of computing $Z_{V}$ using sequential propagators, which will allow us to determine $f_{D_{s}^{*}}$. Since we expect that $Z_{A} / Z_{V} \approx 1$ for our chiral action (for massless fermions $Z_{A} / Z_{V}=1$ ), here we present results for the quantity $\frac{Z_{A} f_{D_{s}^{*}}}{Z_{V} f_{D_{s}}} \approx \frac{f_{D_{s}^{*}}}{f_{D_{s}}}$. 

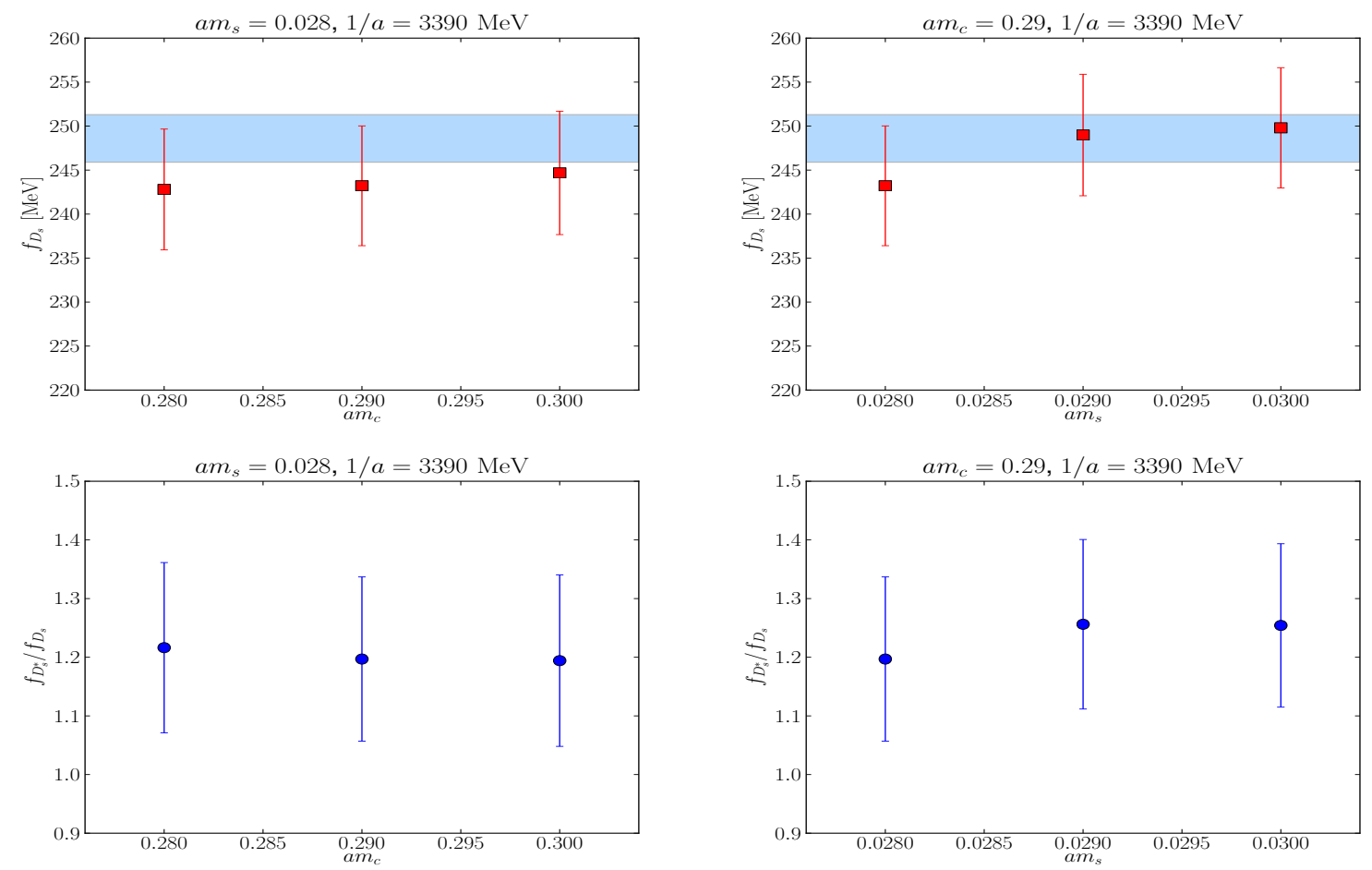

Figure 4: (Top) $f_{D_{s}}$ as a function of charm and strange input masses on the finer of our two ensembles $\left(a^{-1} \approx 3.4 \mathrm{GeV}\right)$. The physical result quoted in the PDG is shown as a blue band. (Bottom) Results for the ratio $f_{D_{s}^{*}} / f_{D_{s}}$, assuming $Z_{A}=Z_{V}$.

The matrix elements in Eqs.(3.1) and (3.2) are determined in the standard way by fitting twopoint correlation functions $\left\langle O^{\dagger}(t) O(0)\right\rangle$ where $O=\left\{A_{4}, V_{4}, P\right\}$. The results presented here are all obtained using Coulomb gauge-fixed point-source propagators. We are also exploring point-wall and wall-wall correlators as well as different combinations of operator (e.g. $A_{4}-P$ ). All uncertainties are computed via single-elimination jackknife.

In Fig. 4, we show results for $f_{D_{s}}$ on the finer of our two ensembles $\left(a^{-1} \approx 3.4 \mathrm{GeV}\right)$ as both the heavy mass $m_{c}$ and light mass $m_{s}$ are varied. We see little variation in the results over the ranges studied, which are consistent with the value given in the PDG. Fig. 4 also shows results for $f_{D_{s}^{*}} / f_{D_{s}}$, where we have assumed that $Z_{A}=Z_{V}$. It is expected that the mixed action effects will be smaller for heavy-light mesons, and in the ratio its effects will be minimal.

\section{Conclusions}

In this work we reported preliminary results on the ground state charmed hadron masses along with charmed-strange meson decay constants by using a mixed action approach, comprising overlap valence quarks, generated on the background of dynamical 2+1+1 flavours HISQ configurations. The results, in particular the hyperfine splitting of $1 \mathrm{~S}$ charmonia, are encouraging and suggest that the overlap valence on $2+1+1$ flavor HISQ configurations is a promising approach to do lattice QCD simulation with light, strange and charm quark together in the same lattice formulation. Discretization errors of the overlap action for the charm quark are reduced by tuning the charm quark mass with kinetic mass, rather than pole mass, as suggested in the Fermilab formulation of heavy quarks [1]. 
This is a continuing study and we expect to be able to do suitable chiral and continuum extrapolations, to make experimentally relevant predictions for various charmed baryons. The splitting $\left(m_{\Omega_{c c c}}-\frac{3}{2} m_{J / \Psi}\right)$, between $J / \Psi$ and the unknown triply-charmed baryon $\Omega_{c c c}$ was found to be $145(10) \mathrm{MeV}$ and 144(10) MeV, on our coarser and finer lattices respectively. We are also studying heavy-light decay constants and in the process of calculating renormalization constants.

\section{Acknowledgement}

The computations were carried on the Blue Gene P of Indian Lattice Gauge Theory Initiative, Tata Institute of Fundamental Research (TIFR), Mumbai. We would like to thank A. Salve and K. Ghadiali for technical support. We are grateful to the MILC collaboration and in particular to S. Gottlieb, for providing us with the HISQ lattices.

\section{References}

[1] A. X. El-Khadra, A. S. Kronfeld and P. B. Mackenzie, Phys. Rev. D 55, 3933 (1997).

[2] S. Aoki et al., Prog. Theor. Phys. 109, 383 (2003); N. H. Christ et al., Phys. Rev. D 76, 074505 (2007); H. -W. Lin and N. Christ, Phys. Rev. D 76, 074506 (2007).

[3] H. Neuberger, Phys. Lett. B417 (1998) 141; ibid. B427 (19998) 353.

[4] A. Bazavov et al. (MILC Collaboration), Phys. Rev. D 87, 054505 (2013); ibid. Phys. Rev. D 82, 074501 (2010); ibid. PoS(Lattice 2010)320 (2010); ibid. PoS(Lattice2012)158.

[5] R. Edwards et al., Phys.Rev. D59 (1999) 094510.

[6] H. Fukaya et al., Phys. Rev. Lett. 98, 172001 (2007); Phys. Rev. D 77, 074503 (2008).

[7] E. Follana et al., Phys. Rev. D75, 054502 (2007); ibid. Nucl. Phys. B (Proc. Suppl.) 129, 447 (2004).

[8] A. Li et al. Phys. Rev. D82 (2010) 114501; N. Mathur et al., PoS LATTICE2010 (2010) 114.

[9] S. Basak, S. Datta, M. Padmanath, P. Majumdar and N. Mathur, PoS LATTICE 2012, 141 (2012).

[10] C.T.H. Davies et al., Phys.Rev. D81 (2010) 034506.

[11] Y. Chen et al., Phys. Rev. D70 (2004) 034502, S.J. Dong et. al, Phys. Rev. Lett. 85 (2000) 5051-5054.

[12] T. Burch et al., Phys.Rev. D81 (2010) 034508; G.C. Donald et al., Phys.Rev. D86 (2012) 094501.

[13] J. D. Bjorken, FERMILAB-CONF-85/69.

[14] M. Padmanath, R. G. Edwards, N. Mathur and M. Peardon, arXiv:1307.7022 [hep-lat].

[15] Y. Namekawa et al. [PACS-CS Collaboration], Phys. Rev. D 87, 094512 (2013).

[16] S. Durr, G. Koutsou and T. Lippert, Phys. Rev. D 86, 114514 (2012).

[17] N. Mathur, R. Lewis and R. M. Woloshyn, Phys. Rev. D 66, 014502 (2002).

[18] R. A. Briceno et al., Phys. Rev. D 86, 094504 (2012).

[19] G. Bali et al., J. Phys. Conf. Ser. 426 (2013) 012017.

[20] M. Padmanath, R. G. Edwards, N. Mathur and M. Peardon, arXiv:1311.4806 [hep-lat].

[21] O. Bar et al., Phys. Rev. D 67, 114505 (2003), ibid. 70, 034508 (2004); J.W. Chen et al., Phys. Rev. D75. 054501 (2007); K. Orginos et al., Phys. Rev. D77, 094505 (2008) and references therein.

[22] M. Lujan et al., Phys.Rev. D86 (2012) 014501. 